\title{
A Receding Horizon Control Approach for Re-Dispatching Stochastic Heterogeneous Resources Accounting for Grid and Battery Losses
}

\author{
Eleni Stai ${ }^{a, *}$, Fabrizio Sossan ${ }^{\mathrm{b}}$, Emil Namor $^{\mathrm{a}}$, Jean Yves Le Boudec $^{\mathrm{a}}$, \\ Mario Paolone ${ }^{\mathrm{a}}$ \\ ${ }^{a}$ École Polytechnique Fédérale de Lausanne (EPFL), Lausanne 1015, Switzerland. \\ ${ }^{b}$ Centre for processes, renewable energies and energy systems (PERSEE), MINES ParisTech, \\ PSL University, 06904, Sophia Antipolis, France.
}

\begin{abstract}
In this paper, we propose a re-dispatch scheme for radial distribution grids hosting stochastic Distributed Energy Resources (DERs) and controllable batteries. At each re-dispatch round, the proposed scheme computes a new dispatch plan that modifies and extends the existing one. To do so, it uses the CoDistFlow algorithm and applies a receding horizon control principle, while accounting for hard time computation constraints that impact on the instantaneous update of a dispatch plan. CoDistFlow handles stochastic DERs and prosumers uncertainties via scenario-based optimization and the non-convexity of the AC Optimal Power Flow by iteratively solving suitably defined convex problems until convergence. We perform numerical evaluations based on real-data, obtained from a real Swiss grid. We show that, with our proposed re-dispatch scheme, the daily dispatch tracking error can decrease more than $80 \%$, even for small battery capacities, and if re-dispatch is frequent enough, it can be eliminated. Finally, we show that redispatch should be performed as often as the market allows and the performance continues to improve.
\end{abstract}

Keywords: Dispatch plan; re-dispatch; intra-day; optimal power flow; grid

\footnotetext{
${ }^{*}$ Corresponding author

Email addresses: eleni.stai@epfl.ch (Eleni Stai), fabrizio.sossan@mines-paristech.fr (Fabrizio Sossan), emil.namor@epfl.ch (Emil Namor), jean-yves.leboudec@epfl.ch (Jean-Yves Le Boudec), mario.paolone@epfl.ch (Mario Paolone)
} 
losses; battery models; scenario-based; power distribution systems; stochastic distributed energy resources;

\section{Introduction \& Contributions}

Stochastic Distributed Energy Resources (DERs) are increasingly deployed in modern power distribution grids. However, due to their uncertain and variable power injections, they can lead to the need of installing and activating large amounts of costly reserves [1]. Jointly dispatching collections of stochastic DERs, loads and controllable batteries may compress the amount of required reserves as it aims at avoiding the scheduling and activation of balancing reserves during realtime operation $[2,3,4]$ and it is advocated, for instance, in Swissgrid's grid code to improve operations reliability [5]. When dispatching, the operator computes a discrete sequence of power values at the Point of Common Coupling (PCC) with the main grid, called the dispatch plan, which is committed with, for instance, the day-ahead electricity market. The dispatch plan can be obtained by solving an Optimal Power Flow (OPF) in which the battery power injections as well as the electrical state of the grid constitute optimization variables. According to [6], in order to compute an efficient dispatch plan, the OPF should account for the uncertainties of DERs and loads, as well as for accurate models of the grid/battery losses and the associated constraints. In real time, while the realization of the uncertain prosumption is being revealed, the batteries are being controlled so as to track the committed dispatch plan $([7,8])$.

However, when tracking a day-ahead dispatch plan during operation, depleted flexibility of the batteries can occur due to the accumulated forecasting error. In this context, it is key to perform intra-day re-dispatching by accounting for new information, i.e., recomputing the dispatch plan for the upcoming time horizons considering updated forecasts of stochastic generation and demand. This solution is promising for further compressing the scheduling and activation of costly primary/secondary frequency control power reserves, thus enabling the integration of more renewable generation. 
In this paper, we propose a re-dispatch scheme for radial distribution grids with stochastic DERs and controllable batteries. Re-dispatch is performed using a receding horizon control $(\mathrm{RHC})$ principle and at regular time intervals, it computes a new dispatch plan that modifies and extends the existing one. At each re-dispatch round, we collect updated information, which consists of (i) the currently observed state-of-energy of the batteries and (ii) updated prosumption forecast scenarios. We use CoDistFlow ([6]) to solve iteratively a scenario-based non-convex OPF problem and compute a new dispatch plan for a number of consecutive time intervals that follow. In order to re-dispatch in a computationally efficient way (i) we had to adapt the receding horizon control principle, and (ii) it was necessary to perform a special design of the optimization problem solved by the CoDistFlow.

The uncertainty of loads and DERs is handled by applying scenario-based optimization. Compared to its alternatives, robust optimization and chanceconstrained optimization, scenario-based optimization has the following advantages: (i) it models properly the uncertainty of stochastic resources [9] (e.g., in a non-parametric way), (ii) it allows for general convex constraints, and (iii) it expresses any existing time correlations. In this work, the scenarios consist of forecasted time-series constructed based on historical and present knowledge.

CoDistFlow is chosen as the most suitable method for repeatedly computing dispatch plans with scenario-based optimization. To the best of our knowledge, CoDistFlow is the only algorithm in the literature for solving a scenario-based OPF so that the solution satisfies the exact power flow equations and the exact grid-security constraints for all the scenarios. As it is shown in [6], existing second-order cone programming OPF relaxation methods that yield exact solutions without scenarios, e.g., $[10,11]$, might not provide exact solutions in case of scenario-based optimization. In addition, common OPF solution approaches that use sequential linearization of the power flow equations with sensitivity coefficients might have a slow convergence and might lead to solutions with nonsatisfactory performance [6]. CoDistFlow converges in few iterations, and the 
numerical evaluations show that it provides a solution with very good performance [6]. This is due to the linearization of the power flow equations that CoDistFlow uses, which is a modified form of Simplified DistFlow so that it is exact around a freely chosen operating point as well as a good approximation under all operating conditions.

We apply a realistic model of battery losses in the context of energy management applications. It represents the internal losses of the grid-connected battery systems using equivalent lossy lines integrated in the power flow model [6]. As opposed to efficiency-based methods, it does not require the use of relaxations or binary variables. By treating charge/discharge losses as grid losses, our model can be integrated directly into the load flow problem and allows us to adopt an accurate representation of batteries' apparent power constraints.

We investigate the importance of re-dispatching by quantifying it through the design of appropriate metrics based on existing reserve markets and by performing evaluations on real data-sets. Specifically, the numerical evaluations are performed on a real-life distribution system, in Switzerland, composed of 34 buses, and the scenarios are constructed with real prosumption data. By solving large-scale optimization problems, we show that by re-dispatching with the proposed scheme, the dispatch-plan tracking error, as well as the corresponding cost, can almost cancel out even for small battery sizes. Moreover, we show how our scheme can be used as a tool to determine the appropriate frequency of re-dispatching given the battery size and conversely.

The rest of the paper is organized as follows. Section 2 summarizes the related literature. Section 3 provides the system model. In Section 4 we describe the proposed re-dispatch approach and in Section 5, we solve the re-dispatch problem. Section 6 presents the evaluation results and finally, Section 7 concludes the paper.

\section{Related Works on Power Distribution Networks Re-dispatch}

Since forecasting errors cannot be avoided, while in some practical cases good forecasts may be unavailable, re-dispatching is an emerging need to increase the 
safe operational margins of the power grid in the presence of intermittent DERs. In the literature, there exist several works that perform re-dispatching for intraday markets and real-time operation of power grids, a synopsis of which is given next.

In [12], the authors propose an intra-day multi-period energy and reserve pre-dispatch model and a real-time single time-step horizon re-dispatch model. Contrary to our approach, prosumption uncertainty is not considered and since the re-dispatch is instantaneous, it does not account for updates in the future forecasts. In the same spirit, [13] performs a single time-step horizon re-dispatching but without considering energy storage systems. The prosumption uncertainties are handled via chance constraints integrated within a second-order cone programming OPF. Comparative evaluations show the benefits for system reliability of accounting for the uncertainty. However, the chance constraints are approximated based on a known parametric probability distribution of the uncertainties, contrary to our proposed approach, which by employing scenario-based optimization, makes no assumptions on their distribution.

RHC is extensively used in the literature for developing dispatching and redispatching schemes. One of the earliest approaches, [14], proposes an RHCbased economic dispatch scheme for a power grid without energy storage, while ignoring grid losses and replacing the uncertain quantities with point forecasts. [15] develops a distributed RHC scheme for storage control in a power grid, without also considering the grid losses or uncertainties. [7] obtains a day-ahead dispatch plan with scenario-based optimization and an RHC-based scheme for real-time control. It neither models the grid nor accounts for the grid constraints. In [16], a real-time RHC-based power control scheme is proposed for a grid with DERs and energy storage using scenario-based optimization for the uncertainty but without modeling the grid. [17] develops a chance-constrained RHC scheme for storage control in the presence of DERs by applying linearizations of the power flow equations with sensitivity coefficients.

Several papers employ RHC-based approaches for controlling microgrids. For 
instance, the schemes proposed in [18] and [19] are based on RHC for operating a microgrid with the aim of minimizing its operating costs. [18] applies scenariobased optimization, whereas [19] replaces the uncertain quantities with point forecasts. In [20], RHC and scenario-based optimization are jointly applied for the operational control of islanded microgrids using the DC approximation of the power flow equations and assuming no energy storage losses. [21] develops a twolayer RHC-based control scheme for a microgrid in two different time-scales. The power grid and the associated constraints are not modeled, whereas uncertainties are considered only in the fast time-scale control via chance constraints. Finally, [22] brings upfront the computational complexity issues of the RHC approaches for economic dispatch and unit commitment and tries to handle them by decoupling the two problems. However, for efficiency purposes, it linearizes the cost functions and it does not model the power grid.

RHC is a powerful tool for controlling power grids while continuously adapting to their evolving, a-priori unknown, state. However, it is computationally demanding, especially in presence of uncertainties, non-convexities and long timehorizons. Thus, existing approaches that use it make simplifications on the grid modeling and the uncertainty modeling, as well as limit the horizon lengths. In this work, we leverage CoDistFlow and we develop a complete RHC-like scheme for intra-day re-dispatching that considers accurate grid modeling, accounts properly for the uncertainty using scenario-based optimization and handles efficiently applicability issues due to computational complexity.

\section{System Model, Battery Model \& Notation}

We consider a balanced and transposed radial distribution grid. The PCC is at index 0 and is assumed to be the slack bus. In view of these working hypotheses, distribution lines and, in general, branches are represented by their single-phase direct-sequence equivalent $\pi$ models (Figure 1(a)). Note that the same model can be used to represent other devices connected between nodes (e.g., series voltage 
regulating transformers $)^{1}$. The node at the top of line $\ell$ closer to the PCC, is denoted as $\operatorname{up}(\ell)$, and the node at the bottom as $\ell$ (Figure 1(a)). We assume, without loss of generality, that there is only a single bus - the bus with index 1 - connected to the PCC. The $N \times N$ matrix $\mathbf{G}$ is the adjacency matrix of the oriented graph of the network excluding the PCC, i.e., $\mathbf{G}_{k, \ell}=1$ for two buses $k, \ell \neq 0$, if $k=\operatorname{up}(\ell)$, otherwise $\mathbf{G}_{k, \ell}=0$.

On each line $\ell$ (Figure $1(\mathrm{a})$ ), let (i) $S_{\ell}^{d}(t)=P_{\ell}^{d}(t)+\jmath Q_{\ell}^{d}(t)$ be the direct sequence complex power fed from the bus $\operatorname{up}(\ell)$ at time $t$ and for scenario $d$, (ii) $z_{\ell}=r_{\ell}+\jmath x_{\ell}$ and $b_{\ell}$ be the direct sequence longitudinal impedance and shunt susceptance of the branch $\ell$, (iii) $f_{\ell}^{d}(t)$ be the square magnitude of the direct sequence current flowing through $z_{\ell}$ at time $t$ and for scenario $d$. $\bar{I}_{\ell}$ is the ampacity limit of branch $\ell$. On each bus/node $i$ (Figure 1(a)), (i) $v_{i}^{d}(t)$ is the square magnitude of the direct sequence voltage at time $t$ and for scenario $d$, (ii) $\underline{v}, \bar{v}$ are lower and upper bounds on $v_{i}^{d}(t)$, i.e., $\underline{v}^{2} \leq v_{i}^{d}(t) \leq \bar{v}^{2}$, (ii) $s_{i}^{d}(t)=p_{i}^{d}(t)+\jmath q_{i}^{d}(t)$ is the complex power injection without the battery power injections $\left(p_{i}^{d}(t)>0, q_{i}^{d}(t)>0\right.$ indicate consumption) at time $t$ and for scenario $d$.

$S^{D P}(t)=P^{D P}(t)+\jmath Q^{D P}(t)$ stands for the dispatched complex power at PCC, at time $t$. At PCC the voltage is assumed fixed, $v_{0}^{d}(t)=1 \mathrm{pu}, \forall t, d$.

Battery Model. In order to account for the internal losses of a battery, we employ the resistance-based model in [6]. The model captures injection and extraction losses through equivalent circuit models ${ }^{2}$. This model is more realistic than, for instance, the commonly adopted constant efficiency-based one, that fails in capturing the nonlinear power-dependent losses of all the components of the

\footnotetext{
${ }^{1}$ We remind that taking into account shunt elements is particularly suitable for underground cables used in urban contexts.

${ }^{2}$ For the power converter (with which the battery is interfaced to the grid), the circuit model is a Norton or Thevenin one depending on the converter's operation as grid-following or grid-forming, respectively. For the battery a series resistance is added to model its losses as described in the following.
} 
power chain (i.e., battery, power converter, and step-up transformer) due to their internal impedance. The adopted equivalent circuit model inherently accounts for this and is particularly appealing since it can be seamlessly integrated into the load flow problem with a virtual node and a virtual line. The battery model is illustrated in Figure 1(b). Specifically, for a battery interfaced to bus $i$ via power conversion devices: (i) a new virtual bus, $\ell$, is added and connects to bus $i$ via a virtual purely resistive line, $\ell\left(z_{\ell}=r_{\ell}, x_{\ell}=0\right.$ ), where $i=u p(\ell)$, (ii) we connect a lossless battery with same capacity and rated power to the virtual bus $\ell$, and (iii) we connect a reactive-power resource to bus $\operatorname{up}(\ell)$. Therefore, we represent a battery (in this case supposed to be interfaced with a grid following converter that has negligible losses) by two controllable resources, namely, the lossless battery at virtual bus $\ell$ and the reactive-power resource at bus up $(\ell)$. Moreover, the internal losses of the battery are equal to the power losses on the purely resistive line and the state-of-energy ( $\mathrm{SoE}$ ) of the battery is equal to the one of the lossless battery. In the Appendix, we explain how the resistance of the newly added virtual line can be assessed experimentally and we provide an experimental validation of the resistance-based battery model. Note that we impose neither ampacity constraints to the virtual line, nor voltage constraints at the virtual node, because both are only part of the battery model. For a battery at virtual bus $\ell$, we define

- $\operatorname{SoE}_{\ell}^{d}(t)$ the state-of-energy at time $t$ and for scenario $d$,

- $\overline{\mathrm{SoE}}_{B, \ell}$ the energy capacity, i.e., $0 \leq \operatorname{SoE}_{B, \ell}^{d}(t) \leq \overline{\operatorname{SoE}}_{B, \ell}$,

- $p_{B, \ell}^{d}(t)$ the charging $\left(p_{B, \ell}^{d}(t) \geq 0\right)$ or discharging $\left(p_{B, \ell}^{d}(t) \leq 0\right)$ power at time $t$ and scenario $d$, without including the battery losses,

- $q_{B, \mathrm{up}(\ell)}^{d}(t)$ the reactive power of the reactive power source,

- $s_{B, \mathrm{up}(\ell)}^{R}$ the battery's converter rated power.

If there exist $N_{B}$ batteries in the grid, the total number of buses increases to $N+N_{B}$ and the admittance matrix is updated accordingly. Finally, after representing all batteries with their models, the battery capacity is non-zero only at the virtual nodes i.e., those within the set $\left\{N+1, \ldots, N+N_{B}\right\}$. 
Collective Notation. We define $P^{d}(t)=\left[P_{1}^{d}(t), P_{2}^{d}(t), \ldots, P_{N}^{d}(t)\right]^{T}$ the active power flow values for all lines. Similarly, we define the vectors: $Q^{d}(t)$ for the reactive power flows, $v^{d}(t)$ for buses' voltage square magnitude and $p^{d}(t), q^{d}(t), p_{B}^{d}(t)$, $q_{B}^{d}(t)$, for the bus active and reactive prosumption injections and battery active and reactive power values, respectively. In Section 5.1, for the needs of CoDistFlow, we use the correction terms $\hat{p}_{\ell}^{d}(t), \hat{q}_{\ell}^{d}(t), \hat{v}_{\ell}^{d}(t)$ and the approximation terms $\tilde{v}_{\ell}^{d}(t)$ (for line $\ell$, scenario $d$ and time $t$ ). Furthermore, $\hat{P}^{d}(t)=\left[\hat{p}_{1}^{d}(t), \ldots, \hat{p}_{N}^{d}(t)\right]^{T}$, $\hat{Q}^{d}(t)=\left[\hat{q}_{1}^{d}(t), \ldots, \hat{q}_{N}^{d}(t)\right]^{T}, \hat{V}^{d}(t)=\left[\hat{v}_{1}^{d}(t), \ldots, \hat{v}_{N}^{d}(t)\right]^{T}$, and $\tilde{V}(t, d)=\left[\tilde{v}_{1}^{d}(t), \ldots, \tilde{v}_{N}^{d}(t)\right]^{T}$. We introduce more compact notations for the electrical state of the grid $E(t, d)$, the corrections $C(t, d)$, the load injections $s(t, d)$ and the battery power values $s_{B}(t, d)$, as $E(t, d)=\left[P^{d}(t) ; Q^{d}(t) ; v^{d}(t)\right], C(t, d)=\left[\hat{P}^{d}(t) ; \hat{Q}^{d}(t) ; \hat{V}^{d}(t)\right]$, $s(t, d)=\left[p^{d}(t) ; q^{d}(t)\right], s_{B}(t, d)=\left[p_{B}^{d}(t) ; q_{B}^{d}(t)\right]$. To conclude: $S$ denotes complex power flows for all lines, scenarios and times, $v$ denotes voltage square magnitude for all buses, scenarios and times, $S^{D P}$ stands for the dispatched complex power for all times, and $f$ for the current square magnitude through the longitudinal impedances for all lines, scenarios and times.

\section{The Re-dispatch Approach}

A dispatch plan is a sequence of $T \in \mathbb{N}$ complex power values at the PCC, corresponding to $T$ consecutive time intervals of duration $\Delta t$ each, i.e, a dispatch plan computed at time $\tau$ is $\left\{S^{D P}(\tau), S^{D P}(\tau+1), \cdots, S^{D P}(\tau+T-1)\right\}$. T is called horizon of the dispatch plan. The dispatch plan is computed ahead of time, i.e., before the realization is revealed, by solving a non-convex OPF; the details of this computation are given in Section 5 .

Our proposed re-dispatch approach is as follows. Every $R$ time intervals, we compute a new dispatch plan, which modifies and extends the existing dispatch plan. It is based on the most recent SoE of the batteries and the most recent prosumption forecasts. This computation cannot be done instantly and new values of the dispatch plan require a delay before being committed. Therefore, we use a time margin $T_{\text {fixed }}$, which upper bounds the time required to compute the new dispatch plan; then, for the next $T_{\text {fixed }}$ time intervals, we impose that the new 
dispatch plan uses the values inherited from the previous dispatch plan. Thus, although the computation of the new dispatch plan has not been yet completed, the values for the next $T_{\text {fixed }}$ time intervals are already known and can be fed to the real-time algorithm that controls the grid operation. Specifically, given an earlier dispatch plan, $\left\{S^{D P}(\tau), S^{D P}(\tau+1), \cdots, S^{D P}(\tau+T-1)\right\}$, a re-dispatch performed at time $\tau+R$ is the computation of a new dispatch plan $\left\{S^{\prime} D P(\tau+R), S^{\prime} D P(\tau+\right.$ $\left.R+1), \cdots, S^{\prime} D P(\tau+R+T-1)\right\}$, subject to the constraints $S^{\prime} D P(\tau+R)=$ $S^{D P}(\tau+R), S^{\prime D P}(\tau+R+1)=S^{D P}(\tau+R+1), \cdots, S^{\prime} D P\left(\tau+R+T_{\text {fixed }}-1\right)=$ $S^{D P}\left(\tau+R+T_{\text {fixed }}-1\right)$. We assume that $T_{\text {fixed }}+R \leq T$ and $T_{\text {fixed }} \leq R$.

Figures 2 and 3 illustrate the non-trivial interplay of successive re-dispatches. Observe that, since re-dispatch is performed after $R$ intervals, the last $T-R-$ $T_{\text {fixed }}$ dispatch plan values are tentative. Even though such values will not be implemented, it is beneficial to compute them, i.e., to have $T>R+T_{\text {fixed }}$. This is because the presence of storage in the grid introduces dependencies across time intervals, which require a sufficient lookahead when computing a dispatch plan.

Our approach differs from the common application of the receding horizon control principle, which implicitly assumes that computation time is negligible and that newly computed setpoints are immediately available. Such an approach is not directly applicable here due to the complexity of solving the AC OPF. An alternative approach would be to compute the new dispatch plan ahead of time in order to allow sufficient time for computation. Specifically, a re-dispatch performed at time $\tau+R$ would be the computation of a new dispatch plan, $\left\{S^{\prime} D P\left(\tau+R+T_{\text {fixed }}\right), S^{\prime} D P\left(\tau+R+T_{\text {fixed }}+1\right), \cdots, S^{\prime} D P\left(\tau+R+T_{\text {fixed }}+T-1\right)\right\}$, which will be deployed at time $\tau+R+T_{\text {fixed }}$. This would be much less accurate because the SoE of the battery at time $\tau+R+T_{\text {fixed }}$ is not yet observable.

\section{Solving the Re-dispatch with CoDistFlow}

In this section, we focus on the efficient computation of the dispatch plan at each re-dispatch round. To do so, we formulate and solve a scenario-based non-convex AC OPF for a radial distribution network with stochastic renewable energy sources and battery storage. As mentioned in the introduction, (i) 
scenario-based optimization handles the prosumption uncertainty, and (ii) we solve the non-convex AC OPF using the CoDistFlow algorithm [6] that provides a solution satisfying the nonlinear power flow equations and the security constraints. The scenarios are constructed based on probabilistic forecasts [9] (see Section 6.2 for details), which are obtained using historical data and present knowledge at the time the OPF is solved. First, we formulate the AC OPF that is solved at $\tau$. We consider the following constraints. Note that $t$ is the time index and $t \in\{\tau, \tau+1 \cdots, \tau+T-1\}$.

Power Flow Equations $\forall d, \forall \ell, \forall t$.

$$
\begin{aligned}
P_{\ell}^{d}(t) & =\sum_{k: \mathbf{G}_{\ell k}=1} P_{k}^{d}(t)+p_{\ell}^{d}(t)+p_{B, \ell}^{d}(t)+r_{\ell} f_{\ell}^{d}(t), \\
Q_{\ell}^{d}(t) & =\sum_{k: \mathbf{G}_{\ell k}=1} Q_{k}^{d}(t)+q_{\ell}^{d}(t)+q_{B, \ell}^{d}(t)-\left(v_{\mathrm{up}(\ell)}^{d}(t)+v_{\ell}^{d}(t)\right) b_{\ell} / 2+x_{\ell} f_{\ell}^{d}(t), \\
f_{\ell}^{d}(t) & =\left\|S_{\ell}^{d}(t)+j \frac{v_{\mathrm{up}(\ell)}^{d}(t) b_{\ell}}{2}\right\|^{2} / v_{\mathrm{up}(\ell)}^{d}(t) .
\end{aligned}
$$

Voltage Constraints, $\forall d, \forall \ell, \forall t$.

$$
\begin{aligned}
& v_{\ell}^{d}(t)=v_{\mathrm{up}(\ell)}^{d}(t)-2 \Re\left\{z_{\ell}^{*}\left(S_{\ell}^{d}(t)+j v_{\mathrm{up}(\ell)}^{d}(t) \frac{b_{\ell}}{2}\right)\right\}+\left\|z_{\ell}\right\|^{2} f_{\ell}^{d}(t), \\
& v_{\mathrm{up}(1)}^{d}(t)=1, \underline{v}^{2} \leq v_{\ell}^{d}(t) \leq \bar{v}^{2} .
\end{aligned}
$$

Ampacity Constraints, $\forall d, \forall \ell, \forall t$.

$$
\begin{aligned}
& \frac{\left(P_{\ell}^{d}(t)\right)^{2}+\left(Q_{\ell}^{d}(t)\right)^{2}}{v_{\mathrm{up}(\ell)}^{d}(t)} \leq \bar{I}_{\ell}^{2}, \\
& \frac{\left(P_{\ell}^{d}(t)-r_{\ell} f_{\ell}^{d}(t)\right)^{2}+\left(Q_{\ell}^{d}(t)-x_{\ell} f_{\ell}^{d}(t)+\frac{\left(v_{\mathrm{up}(\ell)}^{d}(t)+v_{\ell}^{d}(t)\right) b_{\ell}}{2}\right)^{2}}{v_{\ell}^{d}(t)} \leq \bar{I}_{\ell}^{2} .
\end{aligned}
$$

Battery Constraints, $\forall d, \forall \ell, \forall t$.

$$
\begin{aligned}
& \operatorname{SoE}_{B, \ell}^{d}(t+1)=\left(1-\alpha_{D}\right) \operatorname{SoE}_{B, \ell}^{d}(t)+p_{B, \ell}^{d}(t) \Delta t, \\
& a_{B} \overline{\operatorname{SoE}}_{B, \ell} \leq \operatorname{SoE}_{B, \ell}^{d}(t) \leq\left(1-a_{B}\right) \overline{\operatorname{SoE}}_{B, \ell}, \\
& \left(P_{\ell}^{d}(t)\right)^{2}+\left(q_{B, \mathrm{up}(\ell)}^{d}(t)\right)^{2} \leq\left(s_{B, \mathrm{up}(\ell)}^{R}\right)^{2}, \\
& \operatorname{SoE}_{B, \ell}^{d}(0)=\operatorname{SoE}_{B, \ell}^{I},
\end{aligned}
$$

where (i) $0 \leq a_{B} \leq 1$ is a constant parameter used to define a margin on the SoE lower and upper bounds, (ii) $\operatorname{SoE}_{B}^{I}$ is a vector with dimension $N+N_{B}$ and its 
element, $\operatorname{SoE}_{B, \ell}^{I}$, is the observed $\mathrm{SoE}$ of the battery at bus $\ell$ at time $\tau$, and (iii) $\alpha_{D}$ is the coefficient of self-discharge during an interval with duration $\Delta t . \alpha_{D}$ is computed so that the battery self-discharges by $1 \%$ during a day, i.e., $\alpha_{D}=\frac{0.01 \Delta t}{24}$ [23]. Eq. (10) expresses the battery apparent power constraint. It considers the battery active power including the battery losses, since $P_{\ell}^{d}(t)=p_{B, \ell}^{d}(t)+f_{\ell}^{d}(t) \cdot r_{\ell}$. Eq. (10) is more accurate than the corresponding constraint in [6] that does not consider the battery losses. Note that we do not consider terminal constraints on the SoE. This is not required for our re-dispatch scheme, where the dispatch plan horizon $T$ is fixed and higher than the duration of the re-dispatch round $R$. Obviously, the terminal $\mathrm{SoE}$ at one re-dispatch round becomes an intermediate one for the next re-dispatch round.

Fixed Dispatch Plan Constraints: We denote with $S_{\text {fixed }}^{D P}$ a vector of length $T_{\text {fixed }}$ with elements the dispatch plan values inherited from the dispatch plan of the previous re-dispatch round (Section 4). Then, we introduce the following constraints

$$
S^{D P}(\tau)=S_{\text {fixed }}^{D P}(1), S^{D P}(\tau+1)=S_{\text {fixed }}^{D P}(2), \ldots S^{D P}\left(\tau+T_{\text {fixed }}-1\right)=S_{\text {fixed }}^{D P}\left(T_{\text {fixed }}\right),
$$

which will be included in the OPF in order to assign the inherited dispatch plan values to the $T_{\text {fixed }}$ first entries of the new dispatch plan.

The set of constraints (1) - (12) is non-convex due to the Eqs. (3) and (6)-(7). In addition, we define the following objective function:

$$
\begin{aligned}
& w_{1} \sum_{d, t, \ell} \lambda_{d} \max \left(\underline{\mathrm{E}}_{B, \ell}-\operatorname{SoE}_{B, \ell}^{d}(t), 0, \operatorname{SoE}_{B, \ell}^{d}(t)-\overline{\mathrm{E}}_{B, \ell}\right)+w_{2} \sum_{d, t} \lambda_{d}\left|Q_{1}^{d}(t)\right|+w_{3} \sum_{d, t} \lambda_{d}\left|P_{1}^{d}(t)\right| \\
& +w_{4} \sum_{d, t} \lambda_{d} P_{1}^{d}(t)+w_{5} \sum_{d, t} \lambda_{d}\left(\left|P_{1}^{d}(t)-P^{D P}(t)\right|+\left|Q_{1}^{d}(t)-Q^{D P}(t)\right|\right)
\end{aligned}
$$

which is quite generic including diverse sub-objectives weighted by the nonnegative constants $w_{i}, i=1, \ldots, 5$. The first sub-objective encourages the battery SoE to lie withing the preferred range $\left[\underline{\mathrm{E}}_{B, \ell}, \overline{\mathrm{E}}_{B, \ell}\right]$, where $a_{B} \overline{\mathrm{SoE}}_{B, \ell} \leq \underline{\mathrm{E}}_{B, \ell} \leq$ $\overline{\mathrm{E}}_{B, \ell} \leq\left(1-a_{B}\right) \overline{\mathrm{SoE}}_{B, \ell}$. Due to the constraint (9), a feasible battery SoE will lie in $\left[a_{B} \overline{\mathrm{SoE}}_{B, \ell},\left(1-a_{B}\right) \overline{\mathrm{SoE}}_{B, \ell}\right]$, but possibly outside $\left[\underline{\mathrm{E}}_{B, \ell}, \overline{\mathrm{E}}_{B, \ell}\right]$. If the SoE is outside $\left[\underline{\mathrm{E}}_{B, \ell}, \overline{\mathrm{E}}_{B, \ell}\right]$ the objective function increases by the corresponding penalty given by the first term. The second sub-objective minimizes the reactive power at the PCC $\left(\left|Q_{1}^{d}(t)\right|\right)$ that serves the purpose of maximizing the power factor at 
the PCC. The combination of the third and the fourth sub-objectives minimizes the cost of the power exchanged with the main grid. Indeed, $w_{4}-w_{3}$ can be interpreted as the price received when exporting power to the upstream power grid and $w_{3}+w_{4}$ as the price paid when importing power to the local distribution grid from the upstream grid. The fifth sub-objective minimizes the deviation of the realized slack-bus power for each scenario from the dispatch plan value. A high enough positive value should be assigned to $w_{5}$ in order to derive an optimal dispatch plan that, with high probability, will be followed in the real time operation with a small regulation error (e.g., if participating at the market with our feeder as the dispatchable resource).

Note that the Distribution System Operator (DSO) will adjust the weights of the sub-objectives based on each one's importance in the specific application of interest. For instance, in our evaluations, where the purpose is to compute dispatch plans, we set $w_{5}$ to be much higher value than the remaining weights. Weights can be also zero, in which case the corresponding sub-objectives cancel out. Finally, the objective function is a weighted average over all scenarios, where the weight $\lambda_{d}$ is the probability of occurrence of scenario $d$, with $\sum_{d} \lambda_{d}=1$.

The non-convex AC OPF is formulated as follows:

$$
\min _{S, p_{B}, q_{B}, v, S^{D P}, f}(13), \text { s.t. }(1)-(12) \forall t, d, \ell \text {. }
$$

At each re-dispatch round, we compute an updated $S^{D P}$ by solving the nonconvex problem (14) in an efficient way, using CoDistFlow. When solving (14) we should account for the most recently observed batteries' SoE and the most recently computed prosumption scenarios so that the derived dispatch plan adapts to the current information better than the previously computed one. Next, for completeness purposes, we briefly describe CoDistFlow.

\subsection{CoDistFlow}

CoDistFlow [6] consists of two modules, namely: Improved DistFlow (iDF) and Load Flow (LF). These two modules are applied sequentially and iteratively until convergence. 
Improved DistFlow (iDF) and Load Flow (LF) Modules. The iDF module is given as $\left[S^{D P}, S^{\prime}, v^{\prime}, p_{B}, q_{B}\right]=\operatorname{iDF}\left(s, C, \tilde{V}, \operatorname{SoE}_{B}^{I}, S_{\text {fixed }}^{D P}\right)$. We use $S^{\prime}, v^{\prime}$ to differentiate the output of iDF from the output of LF. iDF solves a problem similar to (14), but, with the following difference. It introduces the constant correction terms $\hat{p}_{\ell}^{d}(t), \hat{q}_{\ell}^{d}(t), \hat{v}_{\ell}^{d}(t)$ that replace the variables $r_{\ell} f_{\ell}^{d}(t), x_{\ell} f_{\ell}^{d}(t)$ and $\left\|z_{\ell}\right\|^{2} f_{\ell}^{d}(t)$, respectively, in Eqs. (1)-(2), (4) and (7). In addition, iDF introduces the constant approximation terms, $\tilde{v}_{\ell}^{d}(t)$, which replace the voltage square magnitude variables, $v_{\ell}^{d}(t)$, in Eqs. (6)-(7). With given constant values of the correction and approximation terms, $C, \tilde{V}$, and with the above replacements, the problem (14) becomes convex and can be efficiently solved.

The values of the correction and approximation terms are computed/updated by the LF module, by solving a full AC load flow (Eqs. (1)-(4)) for a specific time and scenario. The LF module is given as $[S(t, d), v(t, d), C(t, d), \tilde{V}(t, d)]=$ $\mathrm{LF}\left(p_{B}(t, d), q_{B}(t, d), s(t, d)\right)$. The batteries are also considered as PQ buses with injections computed by iDF at the previous iteration.

CoDistFlow Algorithm. CoDistFlow is given in Algorithm 1. The superscript $(k)$ denotes the iteration $k$ of CoDistFlow and the index $j$ in lines $7-10$ the $j^{\text {th }}$ element of the corresponding vectors. It takes as inputs: (i) the prosumption forecast scenarios that are updated at the time of re-dispatch, $\tau$, (ii) the batteries SoE as observed at time $\tau$, (iii) the inherited dispatch plan values. It outputs the optimal dispatch plan and the battery trajectories. According to [6], at convergence the obtained solution satisfies the exact (AC) power flow equations and the exact operational constraints (i.e., Eqs. (1)-(11)) within the tolerance bounds imposed by the convergence criterion.

\section{Evaluation Results}

We perform numerical evaluations and comparisons of the proposed re-dispatch scheme. We set $\Delta t=0.25 \mathrm{~h}$, following the paradigm of the time-scales used in the energy markets, e.g., [24]. In addition, we set $T=96$, and $R$ variable in the range $8-24$ to assess the impact of the duration of a re-dispatch round on the performance. We compare the proposed re-dispatch scheme, with a scheme 


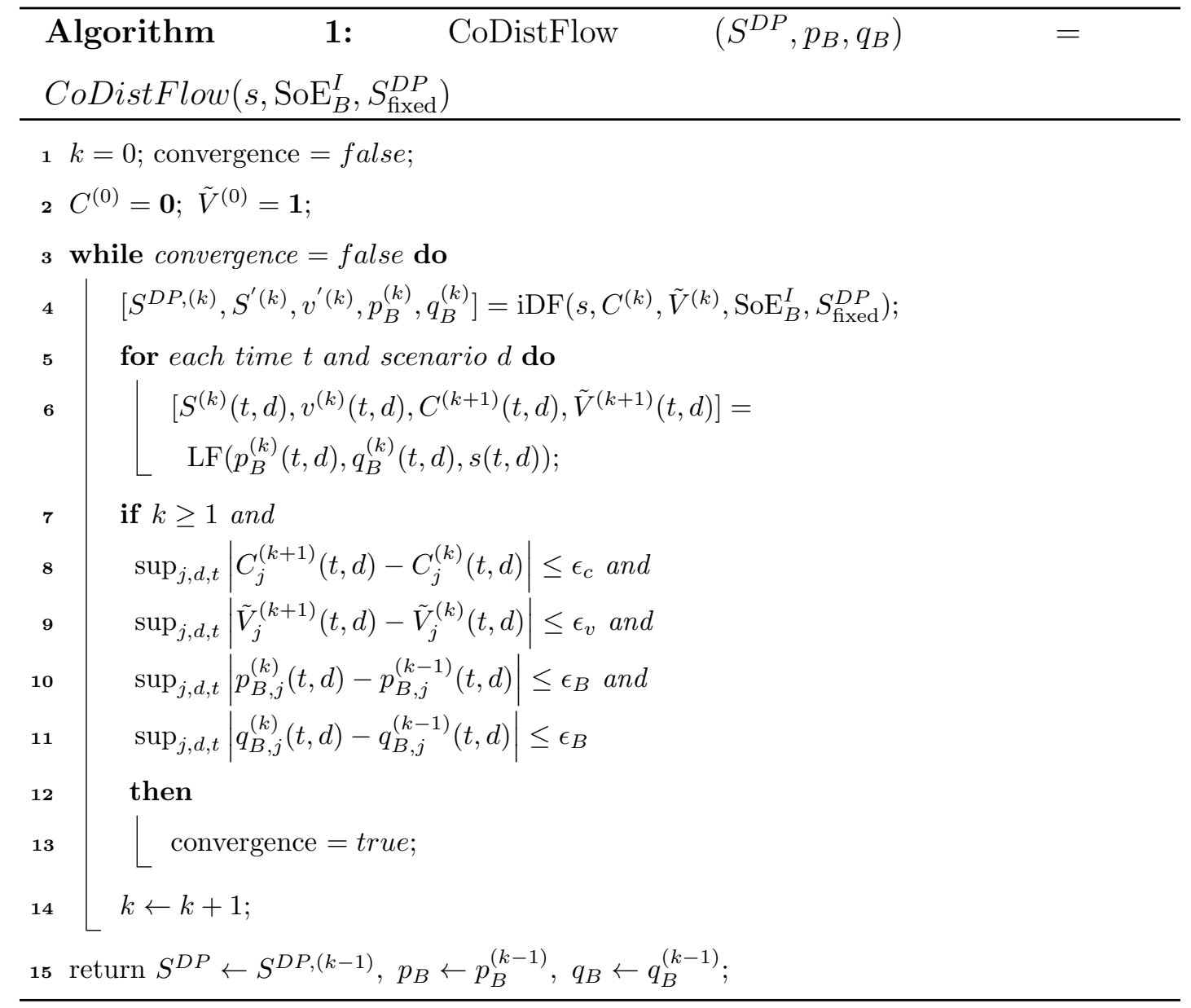


where the dispatch plan is computed just before the day it is applied (i.e., it is not further updated intra-day). We call this scheme "No Re-dispatch".

The simulations are performed for a real Swiss distribution grid, the topology of which is shown in Figure 4. It consists of 34 buses, including the PCC. There is one battery system connected to bus 1, with maximum apparent power 6 MVA and capacity 3 MWh (three-phase), and another battery system connected to bus 23 with the same characteristics. The buses 34 and 35 are virtual buses for the battery models. The base power is $25 \mathrm{MVA}$ and the base voltage is $21 \mathrm{kV}$. We set $\underline{v}=0.9 \mathrm{pu}, \bar{v}=1.1 \mathrm{pu}$, and the initial SoE of all batteries equal to $1 \mathrm{MWh}$, for all scenarios.

We apply $D=80$ scenarios (except when differently mentioned), which have been constructed based on historical data, as described in Section 6.2. Each scenario consists of power prosumption values at all buses, every $\Delta t$, for a $10-$ day period. By choosing this $D$, we accounted for as much detailed information of the uncertainty as possible while maintaining a manageable computational complexity. Note that we tackle large-scale problems (with $N=36, T=96$, $D=80$ and several decision variables per node, time and scenario), with much larger dimensions than in the literature e.g., [20], [16], [18]. We use Matlab with the Yalmip toolbox and the Gurobi solver.

In addition, we assign $w_{1}=w_{2}=w_{3}=w_{4}=w_{6}=1, w_{5}=10$ and $a_{B}=0.1$. $w_{5}$ is chosen much larger than the other weight values since our main objective is dispatchability. Also, $\underline{E}_{B, \ell}=0.15 \cdot \overline{\mathrm{SoE}}_{B, \ell}$ and $\overline{\mathrm{E}}_{B, \ell}=0.85 \cdot \overline{\mathrm{SoE}}_{B, \ell}, \ell \in$ $\{34,35\}$. We set $T_{\text {fixed }}=1$ (except when differently mentioned) since for the grid considered in our evaluations, CoDistFlow takes a few minutes to run at each re-dispatch round. A real-time control algorithm takes battery charge and discharge power decisions every $\Delta t$, aiming to minimize the error in following the dispatch plan. Also, it does not discharge/charge a battery more than 10\%/90\% of its capacity.

\subsection{Evaluation Metrics}

Our primal goal is to provide dispatchability. In the following, we introduce 
our evaluation metrics that quantify how well we achieve this goal. The fifth term in the objective function (13) serves as a proxy of these metrics in the optimization problem solved by CoDistFlow.

Let $P_{1}(t)$ be the realized power at the PCC at time interval $t$, when the true prosumption is revealed and the battery power decisions are applied (after being computed by the real-time control algorithm). The dispatch plan power error at $t$, denoted as $D P_{E}$, is defined as

$$
D P_{E}(t)=P_{1}(t)-P^{D P}(t)
$$

When $D P_{E}(t)=0$, we say that the dispatch plan can be followed at $t$.

Several energy markets such as the Fingrid's one [24] measure power imbalances every $\Delta t=0.25 \mathrm{~h}$, and impose (i) balancing costs on the hourly energy mismatch, and (ii) frequency containment reserve costs for the power imbalance every $\Delta t$. Below, we compute the cost of not following the dispatch plan based on this paradigm. First, we define the per hour energy mismatch at the PCC with respect to the dispatch plan. Let us denote as $t_{h} \in\{0,1,2, \ldots\}$ the index of hours, and assuming that $1 / \Delta t$ is an integer, we define

$$
D E_{E}\left(t_{h}\right)=\sum_{t=t_{h} / \Delta t}^{t_{h} / \Delta t+1 / \Delta t} D P_{E}(t) \Delta t
$$

When $D E_{E}\left(t_{h}\right)>0$, the required energy at hour $t_{h}$ exceeds the planned one and we need to pay up-regulation costs [24], at price $\chi^{+}$. Similarly, when $D E_{E}\left(t_{h}\right)<0$, we pay down-regulation costs, at price $\chi^{-}$. Second, in both cases, we additionally consider the price-to-pay for the frequency containment reserves, denoted as $\chi_{C}$. Thus, the cost at $t_{h}$ is:

$$
D P_{\text {Cost }}\left(t_{h}\right)=\left|\max \left\{\chi^{+} \cdot D E_{E}\left(t_{h}\right), \chi^{-} \cdot D E_{E}\left(t_{h}\right)\right\}\right|+\Delta t \cdot \chi^{C} \sum_{t=t_{h} / \Delta t}^{t_{h} / \Delta t+1 / \Delta t}\left|D P_{E}(t)\right| .
$$


We use Fingrid's market data [24] and, specifically, we apply the average values of the last three months of 2018 equal to $\chi^{+}=56.22 € / \mathrm{MWh}, \chi^{-}=45.97$ $€ / \mathrm{MWh}, \chi^{C}=18.10 € / \mathrm{MWh}$ (this choice is dictated by the impossibility to collect Swiss market data).

Assume $\bar{T}$ hours of grid control in total. Our evaluations are performed for a 10-day period, thus, $\bar{T}=240$. We define the average per day energy mismatch at the PCC, $C D E_{E}$, and the corresponding cost per day, $C D P_{C o s t}$, as

$$
C D E_{E}=\frac{24}{\bar{T}} \sum_{t_{h}=0}^{\bar{T}-1}\left|D E_{E}\left(t_{h}\right)\right|, C D P_{\text {Cost }}=\frac{24}{\bar{T}} \sum_{t_{h}=0}^{\bar{T}-1} D P_{\text {Cost }}\left(t_{h}\right)
$$

\subsection{Day-ahead and Intra-day Forecasts}

This section describes the computation of forecasts and forecast scenarios. Although not a contribution in this paper, their availability is essential for evaluating the performance of the re-dispatch approach. The forecasting engine relies on ARMA models. Their order is chosen by evaluating the partial autocorrelation for the auto-regressive term and autocorrelation for the moving average, following conventional practices for their identification. They are 36 (with nonzero coefficients at lags 1, 24, 25 and 36) and 4. The order of ARMA models at the various buses is the same, but their parameters are estimated for each bus individually. ARMA models are used to generate point predictions for the horizon $T$. Forecasts are updated every 2 hours. The variance of the point predictions is used to build parametric probabilistic forecasts in the form of probability density functions (PDFs); the value of the variance (normalized with respect to the nominal power of each bus) over all buses and all intervals of the horizon lies in the range $0.03 \%-3.2 \%$ per unit.

Forecast scenarios are generated with the method described in [9], briefly summarized hereafter for clarity. It relies on the intuition that, if predicted PDFs are reliable, calculating the values of the PDF for the realizations lead to uniformly distributed series that can be transformed in Gaussian multivariate random variables (i.e., by applying the profit function) and can be tracked by identifying the associated covariance matrix. The covariance matrix is then used 
to generate multivariate Gaussian distributed scenarios with off-the-shelf libraries (e.g., mvnrnd in Matlab). The random sequences are transformed in the final forecast scenarios by, first, applying the inverse probit function and, finally, the inverse predicted PDFs. Temperature is not considered as a regressor as, in this case, there are no electric-thermal loads.

\subsection{Evaluation of the Proposed Re-dispatch Scheme on Real Data Sets}

In this section, we evaluated our re-dispatch scheme for different values of the parameter $R$. The results are shown in Figure 5 and in Table 1.

Table 1 compares the $C D E_{E}$ and $C D P_{C o s t}$ values, as well as the characteristics of the $D E_{E}$ values among all cases. Re-dispatching via our proposed scheme reduces significantly the error in following the dispatch plan in real-time. Specifically, if $R=24, C D E_{E}$ reduces more than 4.5 times. When $R=16, C D E_{E}$ reduces more than 32 times and with $R=8$, it reduces drastically and almost cancels out. Moreover, the $98 \%$ percentile of $D E_{E}$ is drastically reduced; if $R=16$ or lower, it becomes very close to zero. $C D P_{\text {Cost }}$ shows the same trends with $C D E_{E}$. Notice that lack of re-dispatching can lead to significant costs along time and across multiple feeders (here, we account for a single feeder). For instance, in this case, for a single feeder, the expected yearly cost is $24,696 €$ compared to $799.2 €$ that it would have been if we were re-dispatching every 4 hours. If considering that a small city, such as Lausanne with 150,000 inhabitants, has $50-60$ feeders, this cost can reach $1,234,800-1,481,760 €$, which is much more than a cost of $39,960-47,952 €$ if we were re-dispatching with $R=16$.

Table 1: Comparisons of $C D E_{E}, D E_{E}[\mathrm{kWh}], C D P_{\text {Cost }}[€$
\begin{tabular}{|l|l|l|l|}
\hline Scheme & $C D E_{E}$ & $98 \%$ perc. $D E_{E}$ & $C D P_{\text {Cost }}$ \\
\hline No Re-dispatch & 978.8 & 400.91 & 68.6 \\
\hline$R=24$ & 209.53 & 249.51 & 15.57 \\
\hline$R=16$ & 29.95 & 0.0325 & 2.22 \\
\hline$R=8$ & 0.15 & 0.0242 & 0.0095 \\
\hline
\end{tabular}

Figure 5(a) shows the $D P_{E}$ values for all real-time intervals, for schemes without and with re-dispatch. Re-dispatch reduces the number of real-time intervals 
when dispatch-plan tracking fails. In Figure 5(b), we compare the cdf of $D P_{E}$ for all schemes. The maximum value of $D P_{E}$ decreases significantly with re-dispatch: for No Re-dispatch it is equal to $736.39 \mathrm{~kW}$ and for Re-dispatch it is $453.64 \mathrm{~kW}$ with $R=24,405.24 \mathrm{~kW}$ with $R=16$ and $0.365 \mathrm{~kW}$ with $R=8$. Thus, when re-dispatching the required power capacity reserves are smaller.

Finding 1: If we do not re-dispatch, the daily dispatch plan tracking error $\left(C D P_{E}\right)$ and associated cost $\left(C D P_{\text {Cost }}\right)$ may become considerably large, especially at a city level that consists of numerous dispatchable feeders. On the contrary, re-dispatch can accurately track the dispatch plan; if re-dispatching every 6 hours, the daily dispatch plan tracking error and associated cost decrease by around $80 \%$, if re-dispatching every 4 hours they can reduce more than $30 \times$ and if re-dispatching every 2 hours they vanish.

Finding 2: We should re-dispatch as often as the market allows and the $C D P_{\text {Cost }}$ continues to reduce. For example, in the examined grid, it is not worth re-dispatching more frequently than every two hours, since at this frequency $C D P_{\text {Cost }}$ is already almost zero.

In Figs. 5(c)-5(f), for better illustration purposes, we focus on times between $t_{r}=800$ and $t_{r}=960$. Figs. 5(c), 5(d) compare the dispatch plan and the realized power, $P_{1}$, at the $\mathrm{PCC}$ without and with re-dispatch. By comparing Figure 5(a) with Figs. 5(c), 5(d), we observe that the error is just one order of magnitude smaller than the PCC power. Therefore it is important to reduce it. Finally, failures in following the dispatch plan are due to depleting the flexibility of the batteries. Figs. 5(e), 5(f) present the SoE of the batteries at the virtual nodes 34 and 35 . When the batteries are both full and the generation is greater than the consumption, or when they are both empty and the consumption is greater than the generation, there is a failure in following the dispatch plan.

Let us focus on the spike before the time interval 950 in Figure 5(d). This spike is due to three reasons, (i) an increased demand, (ii) for both batteries, the SoE is at the lowest possible level just before the spike (Figure 5(e), 5(f)), and (iii) the battery at the virtual bus 34 starts charging (Figure 5(e)). Therefore, 
since the batteries cannot discharge, the demand should be satisfied by importing power from the main grid. In addition, the imported power further increases in order to charge the battery so as to better accommodate the future grid operation.

Finding 3: Re-dispatch allows for accurately tracking the dispatch plan in real-time, even if it has spikes, i.e., just before the time interval 950 in Figure 5(d). On the contrary, in Figure 5(c) where we do not re-dispatch, the dispatch plan tracking fails for the corresponding time interval although there is no spike.

Next, we vary the number of scenarios, $D$, and study its impact on the time complexity and performance of the proposed re-dispatch scheme. Time complexity refers to the time that CoDistFlow requires to compute an updated $S^{D P}$ at a re-dispatch round and does not depend on $R$. We observe that the time complexity increases with the number of scenarios. In the cases examined, it is less than $10 \mathrm{~min}$; hence we choose $T_{\text {fixed }}=1$ that corresponds to $15 \mathrm{~min}$. As a result, re-dispatching every 2 hours for canceling out errors and costs (Table 1 ) is totally possible as far as time complexity is considered for the examined grid. In general, the error values, $C D E_{E}$, are smaller for larger $D$. However, introducing re-dispatch leads to a much larger improvement in $C D E_{E}$ than increasing the number of scenarios of the No Re-dispatch scheme.

Table 2: Comparisons w.r.t. the number of scenarios.

\begin{tabular}{|l|l|l|l|}
\hline$D$ & $C D E_{E}[\mathrm{kWh}] R=24$ & $C D E_{E}[\mathrm{kWh}]$ No Re-dispatch & Time complexity (min) \\
\hline 5 & 300.44 & 1032 & 0.35 \\
\hline 15 & 236.4 & 996.92 & 1.3 \\
\hline 30 & 234.37 & 992.01 & 4 \\
\hline 50 & 223.78 & 987.41 & 6 \\
\hline 80 & 209.53 & 978.8 & 9 \\
\hline
\end{tabular}

Finally, we vary the value of $T_{\text {fixed }}$. A higher value of $T_{\text {fixed }}$ allows for solving an optimization problem with higher computational complexity at each redispatch round. The computational complexity increases by a significant increase in the number of scenarios. In Figure 6, we observe the following:

- If we re-dispatch every 2 hours (i.e., $R=8$ ), $C D P_{E}$ is almost stable when varying $T_{\text {fixed }}$ from 1 to 6 . In other words, if e.g., the computational complexity 
of updating the dispatch plan is 1.5 hours, re-dispatching every two hours with the proposed re-dispatch scheme shows almost the same performance as if there is a very small computational complexity.

- If we re-dispatch every 4 or 6 hours, $C D P_{E}$ increases almost linearly with $T_{\text {fixed }}$. If the dispatch plan is fixed for the first 2.5 hours (i.e., $T_{\text {fixed }}=10$ ), then the $C D P_{E}$ attains a value double than for $T_{\text {fixed }}=1$.

- In general, the impact of $T_{\text {fixed }}$ on the performance can be characterized as small. Observe that the $C D P_{E}$ for $T_{\text {fixed }}=6$ and $R=8$ is still much lower than the $C D P_{E}$ for $T_{\text {fixed }}=1$ and $R=16$ or 24 . Similarly, the $C D P_{E}$ for $T_{\text {fixed }}=10$ and $R=16$ is much lower than the one for $T_{\text {fixed }}=1$ and $R=24$.

- Re-dispatching more often, if the market allows, is beneficial for all computational complexity values (as long as they are appropriately bounded, i.e., $T_{\text {fixed }}<R$ according to the assumptions in Section 4).

Finding 4 : If re-dispatch is frequent (i.e., $R=8$ ), the performance of our re-dispatch scheme is not affected by the computational complexity of updating the dispatch plan. If re-dispatch is not very frequent (i.e., $R=12$ or 24 ), the performance of our re-dispatch scheme deteriorates linearly with the computational complexity of updating the dispatch plan.

\subsection{Study of the Proposed Re-dispatch Scheme with Varying Battery Size}

In this part, we evaluate the effect of battery size on our scheme. The battery positions remain the same. Figure 7 shows the results. The horizontal axis represents the sum of the two battery sizes. The $C D P_{\text {Cost }}$ values decrease as the battery size increases, since a larger battery provides more flexibility to both the re-dispatch and the real-time control algorithms. The improvement that Redispatch brings compared to No Re-dispatch is even more larger for larger battery capacities. For example, for small battery capacities, re-dispatch with $R=24$ reduces the $C D P_{\text {Cost }}$ (compared to No re-dispatch) by $30 \%-64 \%$, whereas, for larger battery sizes, the reduction reaches up to $80 \%$ or more. In addition, we can make the following important observations.

First, with re-dispatch, $C D P_{\text {Cost }}$ (and $C D E_{E}$ correspondingly) cancels out 
for practical battery sizes, e.g., $3 \mathrm{MWh}$ if $R=8,6 \mathrm{MWh}$ if $R=12$ and $12 \mathrm{MWh}$ if $R=24$. On the contrary, when there is no re-dispatch, $C D P_{\text {Cost }}$ decreases with an extremely small rate with respect to the battery size and thus, its elimination requires unrealistically large battery sizes. Note that the value of $C D P_{C o s t}$ without re-dispatch for a battery size $30 \mathrm{MWh}$ is the same as the value of $C D P_{\text {Cost }}$ with re-dispatch for a battery size $3 \mathrm{MWh}$.

Therefore, re-dispatch, if allowed by the market, should be preferred compared to increasing the battery size. For example, for a battery size of $3 \mathrm{MWh}$, if we introduce re-dispatch every 6 hours, $C D P_{\text {Cost }}$ decreases by around $60 \%$. Without re-dispatch a decrease of $60 \%$ in $C D P_{\text {Cost }}$ can be achieved only by increasing the battery size to $9 \mathrm{MWh}$, i.e., by 3 times. If considering the battery investment cost, which according to [25], is $280 € / \mathrm{kWh}$, the cost for the additional $6 \mathrm{MWh}$ is $1,680,000 €$.

Second, we define as total cost the sum of the $C D P_{\text {Cost }}$ plus the per day battery investment cost. If considering that the typical battery lifetime is 20 years, the per day battery investment cost is equal to $0.038 € / \mathrm{kWh}$ based on [25]. Figure 8 shows the concave form of the total cost versus the battery size. For small battery sizes the $C D P_{\text {Cost }}$ prevails the total cost value, whereas for large battery capacities, battery investment is the prevailing source of cost. Generally, for all schemes, the minimum total cost is achieved for small battery sizes. However, re-dispatch achieves a significant reduction in the minimum total cost compared to No re-dispatch that can exceed $3 \times$.

Finding 5: Canceling dispatch plan errors is not possible without re-dispatch. Specifically, realistic battery sizes cannot remove the dispatch plan error except if we re-dispatch. With re-dispatch, $C D P_{\text {Cost }}$ is eliminated for battery sizes that can be applied in practice. Re-dispatching is less costly and much more efficient than increasing the battery size.

Finding 6 : Re-dispatch can achieve a $3 \times$ smaller minimum total cost (i.e., the sum of the $C D P_{\text {Cost }}$ plus the per day battery investment cost) compared to No re-dispatch. 
Finding 7: Our re-dispatch scheme can serve as a tool for the DSO to determine the optimal frequency of re-dispatch for given battery sizes or the opposite.

\section{Conclusions}

We have proposed and evaluated a re-dispatch scheme for distribution grids with DERs and batteries. It applies a receding horizon, while accounting for computational complexity issues. The update of the dispatch plan is achieved via CoDistFlow, which efficiently accounts for the grid and battery losses and the grid constraints. The evaluations are performed on a real Swiss power distribution grid by using real data. Our re-dispatch scheme can reduce more than $3 \times$ the total cost of operations consisting of the daily dispatch-plan tracking cost and the battery investment cost and it can eliminate the dispatch plan tracking error for practical battery sizes. Moreover, we show that increasing the frequency of re-dispatching should be preferred over increasing the battery size in order to reduce the dispatch plan tracking errors, as it is less costly. To add to this, if we re-dispatch frequently, the performance of our proposed scheme is not affected by the computational complexity of CoDistFlow. Thus, we should re-dispatch as frequently as the market allows in view of the improvement in performance, i.e., re-dispatching every 2 hours is ideal, as at this frequency, the dispatch plan tracking costs cancel out.

\section{Appendix: Validation of the Resistance-based Battery Model}

In this appendix, we explain how we derive the resistance value of the virtual line of the resistance-based battery model by first estimating experimentally the round-trip energy losses of the battery. In addition, we perform an experimantal validation of the model.

Round-trip energy losses of the battery. They are determined within a specific experimental session where the battery is controlled to undergo a series of discharge/charge cycles at different power levels between two predefined state-ofcharge (SoC) values. Note that here we use the SoC instead of the SoE as we use 
measurements and the SoC can be directly measured by the battery management system. An example of the experiments is shown in Figure 9: starting from a resting situation (where voltage and charge dynamics are extinguished), the battery is first discharged at $50 \mathrm{~kW}$ until $25 \%$, and then charged at $50 \mathrm{~kW}$ until the initial SoC level. The discrete sum over time of the battery (charging/discharging) power multiplied by $\Delta t$, which should be zero in a lossless battery, gives the round-trip energy losses. After a resting phase, this process is repeated for different charging/discharging power values and SoC levels.

The experimental round-trip energy losses for the $560 \mathrm{kWh} / 720 \mathrm{kVA}$ Lithiumion battery of EPFL [7] are shown in Figure 10 for different values of the SoC and charge/discharge power. The values are scaled over the energy exchanged during the round-trip cycle, which was $5 \%$ of the energy capacity. Energy losses take their highest values at low charge/discharge powers and show similar trend for the different SoC levels.

Data-driven computation of the resistance. We model the battery as an ideal power source with a series resistance to capture energy losses, as shown in Fig. 1(b). The advantage of this model is that it can be integrated in an optimal power flow problem and capture losses without requiring the use of binary variables to detect charging or discharging conditions. In the following, we omit the scenario index, $d$, for the ease of presentation. The real power output of the battery is $P_{\ell}(t)$. The power losses on the resistance $r_{\ell}$ are:

$$
f_{\ell}(t) r_{\ell}=\left(\frac{P_{\ell}(t)}{v_{u p(\ell)}(t)}\right)^{2} r_{\ell}
$$

As the voltage at the grid node is regulated near $1 \mathrm{pu}$, we assume $v_{u p(\ell)}(t)=1, \forall t$ in Eq. (19). The objective is to design the value of the resistance $r_{\ell}$ such that the accumulated power losses over time amount to the energy losses identified in the experimental session described above. By denoting the measured energy losses as $\Delta E$, this reads as:

$$
\sum_{t=0}^{T}\left(P_{\ell}(t)\right)^{2} r_{\ell} \Delta t=\Delta E
$$

We use the expression above to estimate a constant value of $r_{\ell}$ :

$$
r_{\ell}=\Delta E /\left(\sum_{t=0}^{T} P_{\ell}(t)^{2} \Delta t\right) .
$$


Since there are multiple values of $\Delta E$ resulting from the experiments at various SoC and power values as reported in Figure 9, we choose the median value of $r_{\ell}$ as it was the best performing. Modeling the dependence of $\mathrm{R}$ to the SoC and power levels will be considered in future works.

Experimental validation. Validation results are performed considering measurements from a validation data set, not included in the training data set. The validation effort refers to showing that including the losses estimation in a simple $\mathrm{SoC}$ estimation process is beneficial. When considering losses, the SoC is computed as the integral over time of the charging/discharging power minus the losses on the resistance, all divided by the energy capacity of the battery. When neglecting losses, $r_{\ell}$ equals 0 , and the SoC is the integral over time of the charging/discharging demand divided by the energy capacity of the battery. The comparison between loss-aware and lossless SoC estimations is given in Figure 11 versus the SoC measurements from the battery management system. Loss-aware estimations accurately track the measured SoC, whereas lossless estimations are slowly drifting away from the measured value over time.

\section{References}

[1] D. Pudjianto, C. Ramsay, G. Strbac, Virtual power plant and system integration of distributed energy resources, IET Renewable Power Generation 1 (1) (2007) 10-16. doi:10.1049/iet-rpg:20060023.

[2] R. Lueken, J. Apt, The Effects of Bulk Electricity Storage on the PJM Market, Energy Systems 5 (4) (2014) 677-704.

[3] A. Preskill, D. Callaway, How Much Energy Storage do Modern Power Systems Need?, arXiv preprint arXiv:1805.05115 (2018).

[4] M. Bozorg, F. Sossan, J.-Y. L. Boudec, M. Paolone, Influencing the Bulk Power System Reserve by Dispatching Power Distribution Networks Using Local Energy Storage, Electric Power Systems Research 163 (2018) 270 - 279. doi:https://doi.org/10.1016/j.epsr.2018.06.017.

[5] General balance group regulations, Tech. rep., Swissgrid AG (2019). 
[6] E. Stai, L. Reyes-Chamorro, F. Sossan, J. Y. L. Boudec, M. Paolone, Dispatching Stochastic Heterogeneous Resources Accounting for Grid and Battery Losses, IEEE Transactions on Smart Grid 9 (6) (2018) 6522-6539. doi:10.1109/TSG.2017.2715162.

[7] F. Sossan, E. Namor, R. Cherkaoui, M. Paolone, Achieving the Dispatchability of Distribution Feeders Through Prosumers Data Driven Forecasting and Model Predictive Control of Electrochemical Storage, IEEE Trans. on Sustainable Energy 7 (4) (2016) 1762-1777.

[8] R. Gupta, F. Sossan, E. Scolari, E. Namor, L. Fabietti, C. Jones, M. Paolone, An ADMMbased Coordination and Control Strategy for PV and Storage to Dispatch Stochastic Prosumers: Theory and Experimental Validation, in: Power Systems Computation Conference (PSCC), 2018.

[9] P. Pinson, H. Madsen, H. A. Nielsen, G. Papaefthymiou, B. Klockl, From Probabilistic Forecasts to Statistical Scenarios of Short-Term Wind Power Production, Wind Energy 12 (1) (2009) 51-62.

[10] N. Li, L. Chen, S. H. Low, Exact Convex Relaxation of OPF for Radial Networks Using Branch Flow Model, in: IEEE Int'l Conf. on Smart Grid Com., 2012, pp. 7-12.

[11] M. Nick, R. Cherkaoui, J. L. Boudec, M. Paolone, An Exact Convex Formulation of the Optimal Power Flow in Radial Distribution Networks Including Transverse Components, IEEE Transactions on Automatic Control 63 (3) (2018) 682-697.

[12] Y. Ding, M. Xie, Q. Wu, J. Ostergaard, Development of Energy and Reserve Pre-dispatch and Re-dispatch Models for Real-time Price Risk and Reliability Assessment, IET Generation, Transmission Distribution 8 (7) (2014) 1338-1345. doi:10.1049/iet-gtd.2013.0822.

[13] C. Hamon, M. Perninge, L. Soder, The Value of Using Chance-constrained Optimal Power Flows for Generation Re-dispatch Under Uncertainty with Detailed Security Constraints, in: IEEE PES Asia-Pacific Power and Energy Engin. Conf. (APPEEC), 2013, pp. 1-6. doi:10.1109/APPEEC.2013.6837148.

[14] L. Xie, M. D. Ilic, Model Predictive Dispatch in Electric Energy Systems with Intermittent Resources, in: 2008 IEEE International Conference on Systems, Man and Cybernetics, 2008, pp. 42-47. doi:10.1109/ICSMC.2008.4811248.

[15] K. Baker, J. Guo, G. Hug, X. Li, Distributed MPC for Efficient Coordination of Storage and Renewable Energy Sources Across Control Areas, IEEE Transactions on Smart Grid 7 (2) (2016) 992-1001. doi:10.1109/TSG.2015.2512503. 
[16] P. Patrinos, S. Trimboli, A. Bemporad, Stochastic MPC for Real-time Market-based Optimal Power Dispatch, in: 50th IEEE Conf. on Decision and Control and European Control Conf., 2011.

[17] E. Dall'Anese, K. Baker, T. Summers, Chance-Constrained AC Optimal Power Flow for Distribution Systems With Renewables, IEEE Transactions on Power Systems 32 (5) (2017) 3427-3438.

[18] A. Parisio, E. Rikos, L. Glielmo, A Model Predictive Control Approach to Microgrid Operation Optimization, IEEE Transactions on Control Systems Technology 22 (5) (2014) $1813-1827$.

[19] R. Palma-Behnke, C. Benavides, F. Lanas, B. Severino, L. Reyes, J. Llanos, D. Sáez, A Microgrid Energy Management System Based on the Rolling Horizon Strategy, IEEE Transactions on Smart Grid 4 (2) (2013) 996-1006.

[20] C. A. Hans, P. Sopasakis, A. Bemporad, J. Raisch, C. Reincke-Collon, Scenario-based Model Predictive Operation Control of Islanded Microgrids, in: 54th IEEE Conference on Decision and Control (CDC), 2015, pp. 3272-3277.

[21] S. Raimondi Cominesi, M. Farina, L. Giulioni, B. Picasso, R. Scattolini, Two-layer Predictive Control of a Micro-grid Including Stochastic Energy Sources, in: 2015 American Control Conference (ACC), 2015, pp. 918-923. doi:10.1109/ACC.2015.7170851.

[22] I. Novickij, G. Joos, Model Predictive Control Based Approach for Microgrid Energy Management, 2019, pp. 1-4. doi:10.1109/CCECE.2019.8861781.

[23] S. Delalay, Ph.D. Thesis (2013).

URL https : //infoscience.epfl.ch/record/187365/files/EPFL_TH5768.pdf?version=1, (Accessed: 22/06/2019)

[24] Fingrid, Reserve Market Information (2019).

URL https ://www.fingrid.fi/en/electricity-market/reserves_and_balancing/reservemarket-information, (Accessed: 22/01/2019)

[25] B. Nykvist, M. Nilsson, Rapidly Falling Costs of Battery Packs for Electric Vehicles, Nat. Clim. Change 5 (4) (2015) 329-332. 


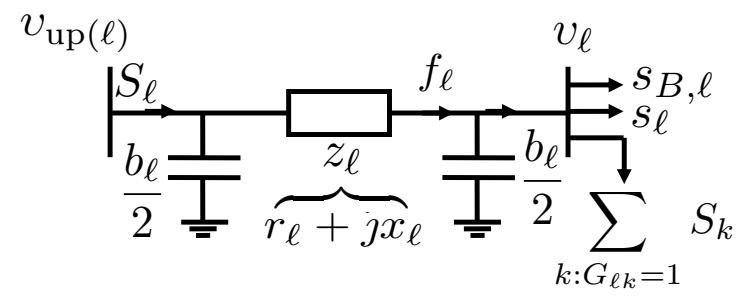

(a) $\pi$ model of a line.

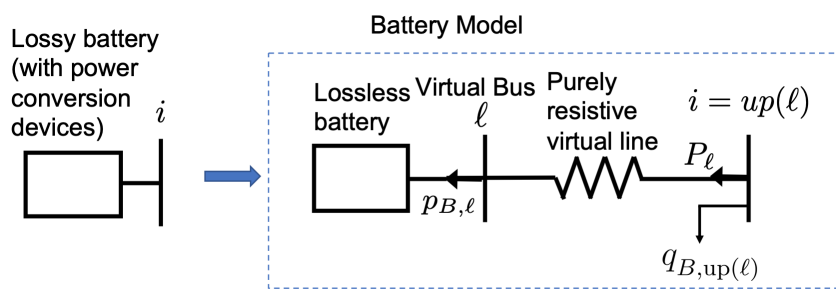

(b) Battery model.

Figure 1: Line (a) and Battery (b) models. 


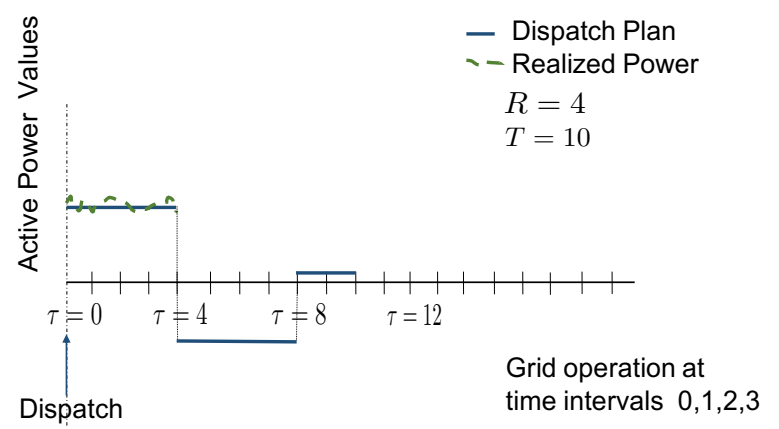

(a) At $\tau=0$, we compute a dispatch plan for a horizon $T=10$ time intervals ahead.

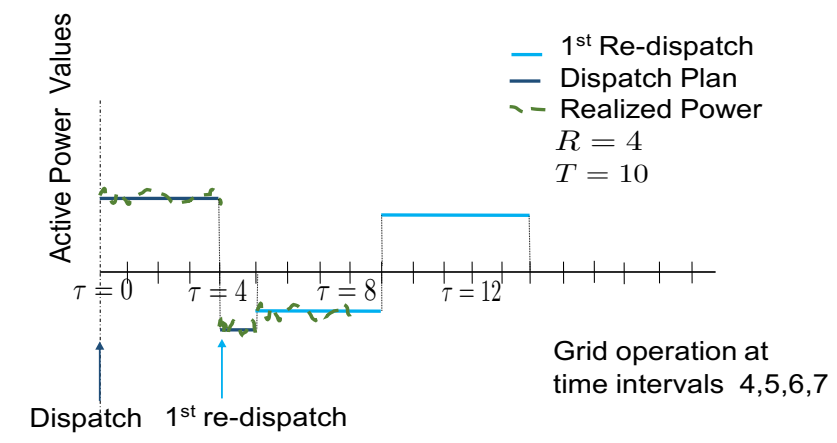

(b) Re-dispatch at time $\tau=4$. We set $T_{\text {fixed }}=1$; the new dispatch plan inherits the value for the time interval 4 from the dispatch plan computed at $\tau=0$.

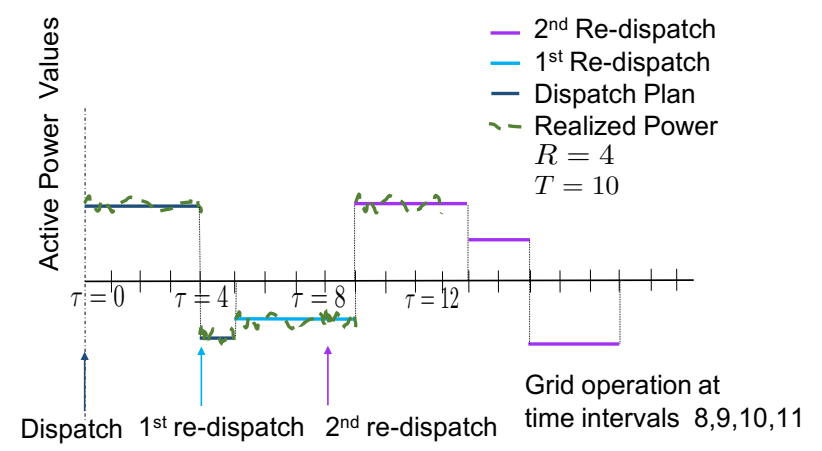

(c) Re-dispatch at time $\tau=8$. The new dispatch plan inherits the value for the time interval 8 from the dispatch plan computed at $\tau=4$.

Figure 2: Illustration of our re-dispatch approach for parameter values $T=10$ and $R=4$. During the grid operation, the real time control algorithm takes battery charge and discharge power decisions in a finer time-scale with the aim of following the dispatch plan (green dotted curly line). 


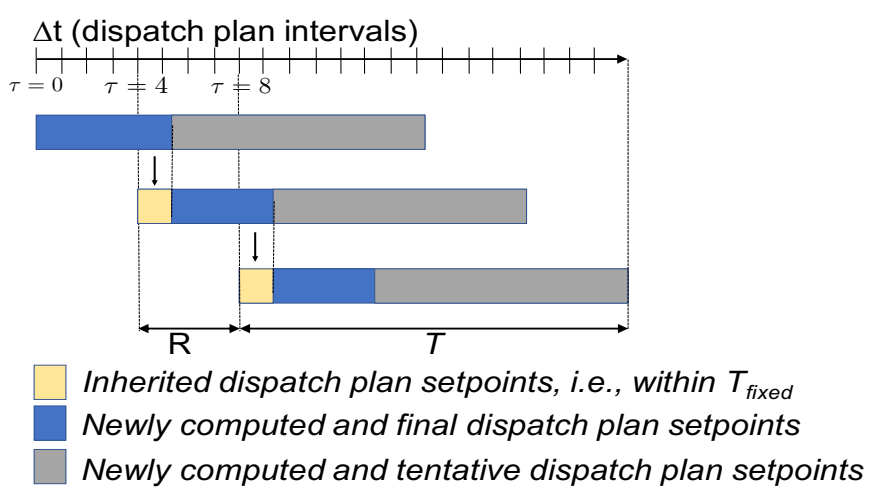

Figure 3: At each re-dispatch round, 1 value is inherited from the previous computation (yellow part of the dispatch plan), 4 values are newly computed and finally applied (blue part of the dispatch plan) and the remaining values are tentative and will be replaced by newly computed ones in the next re-dispatch rounds (grey part of the dispatch plan).

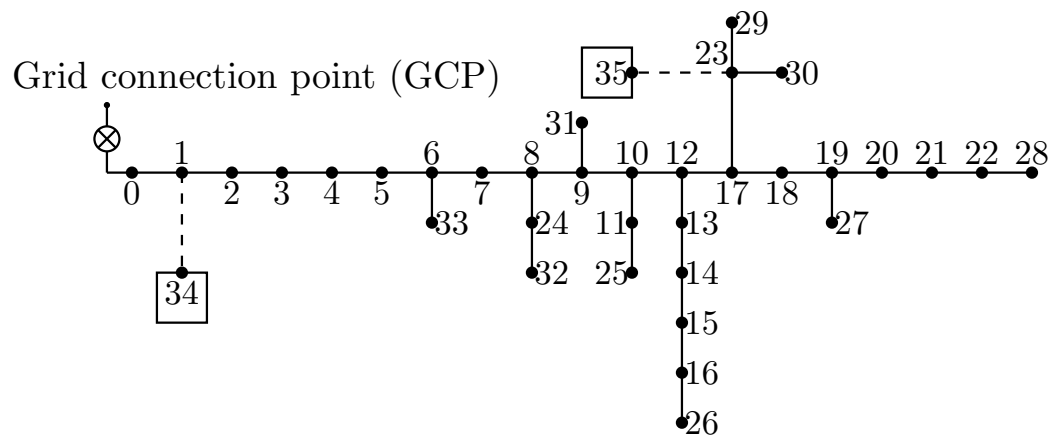

Figure 4: Illustration of the real Swiss grid used for our numerical evaluations. The squares indicate the virtual nodes added for the battery models. The lossy batteries lie at nodes 1 and 23. 


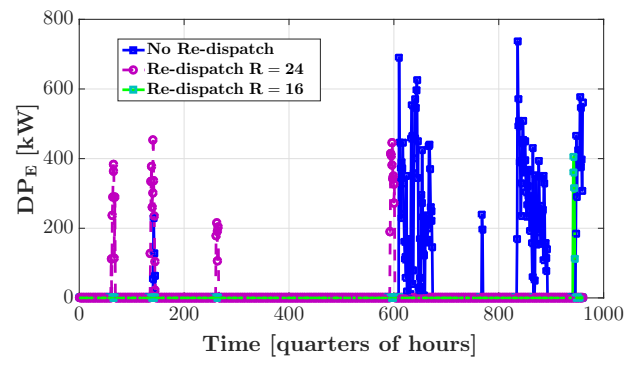

(a) $D P_{E}$ values every 15 minutes.

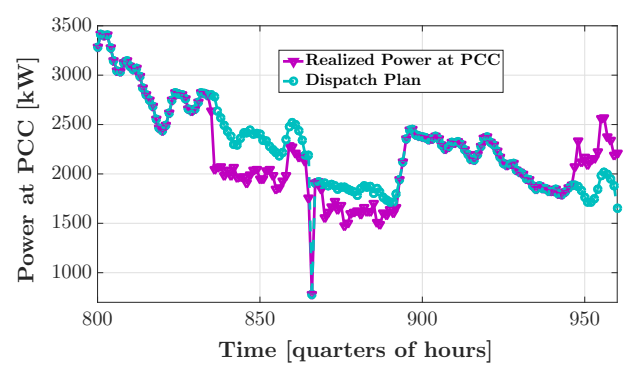

(c) Dispatch plan vs realized power at PCC,

No Re-dispatch (Zoom).

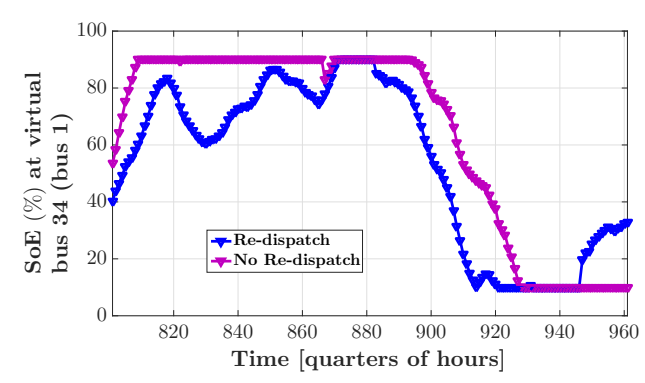

(e) $\operatorname{SoE}(\%)$ at bus 1 (Zoom).

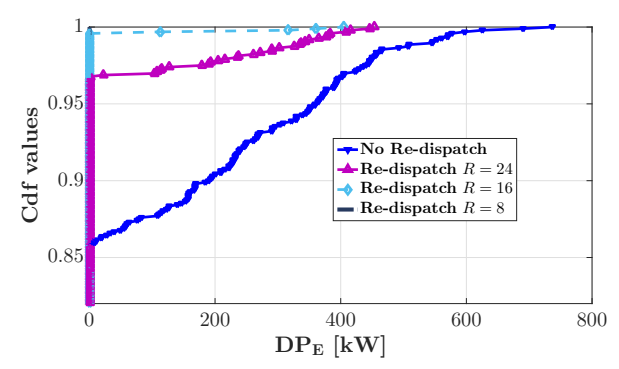

(b) Cdf comparison.

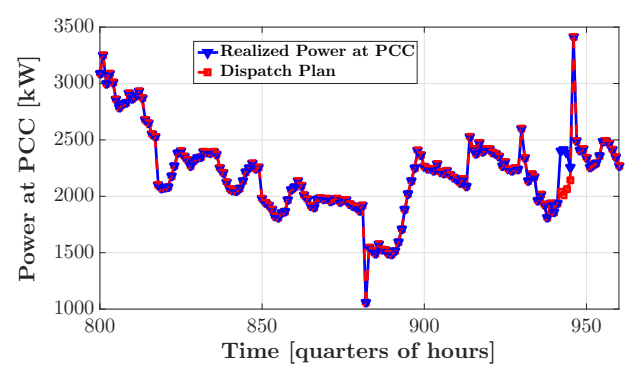

(d) Dispatch plan vs realized power at PCC, Re-dispatch (Zoom).

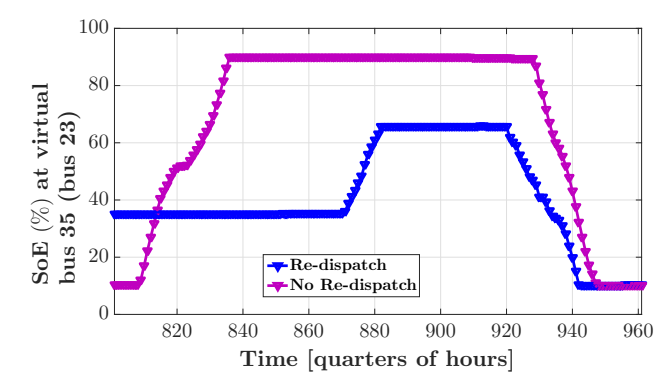

(f) $\mathrm{SoE}(\%)$ at bus 23 (Zoom).

Figure 5: SoE and dispatch plan error comparisons. (a), (b) We observe that if re-dispatching more frequently the errors decrease. However, at the threshold value $R=8$, the errors are negligible; hence, it is not worth to re-dispatch more frequently. (c)-(f) A failure in tracking the dispatch plan emerges when both batteries have a very low or a very high SoE. 


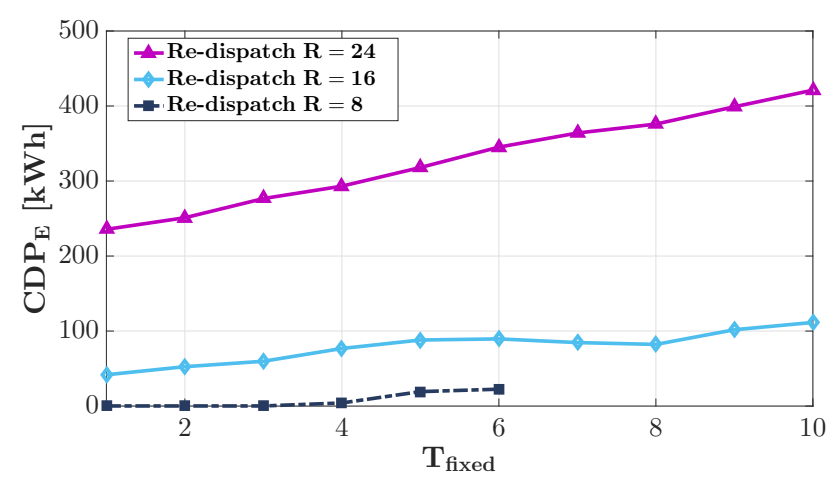

Figure 6: $C D P_{E}$ vs $T_{\text {fixed }}$ for different values of $R$. $T_{\text {fixed }}$ corresponds to the time computational complexity of updating the dispatch plan at a re-dispatch round.

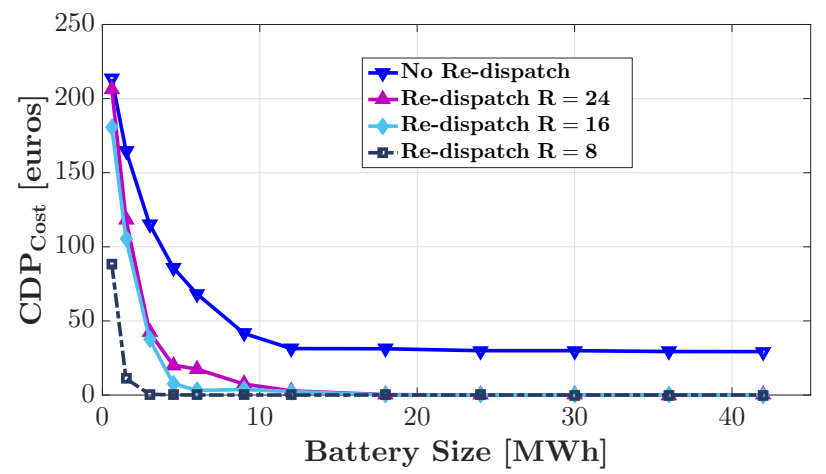

Figure 7: $C D P_{\text {Cost }}$ vs battery size. When re-dispatching the cost cancels out for practical battery sizes.

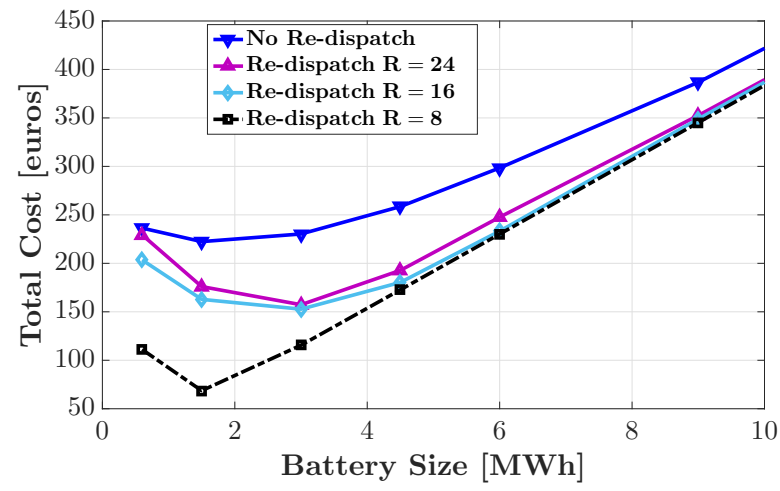

Figure 8: Total cost equal to the sum of the $C D P_{\text {Cost }}$ and the per day battery investment cost. 


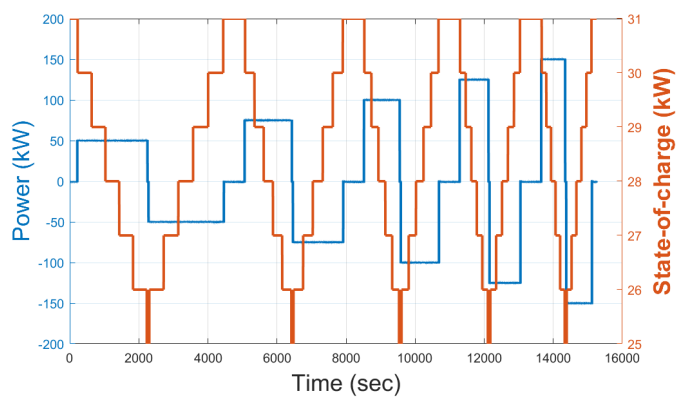

Figure 9: Measured active power at the output of the battery's power converter and battery's SoC during the experiments to determine the round-trip energy losses.

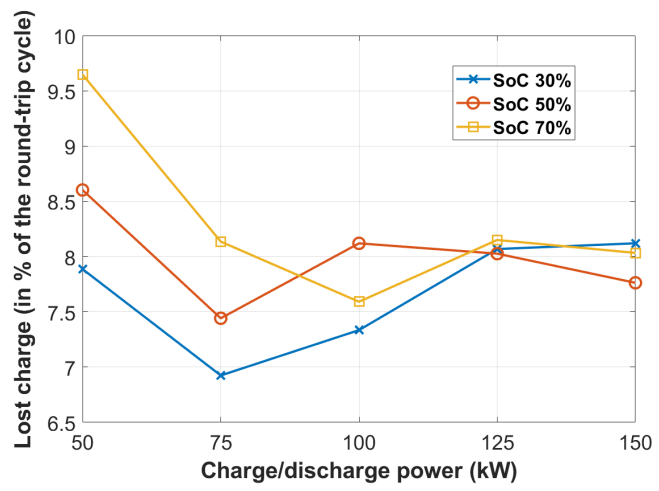

Figure 10: Experimental round-trip energy losses for the $560 \mathrm{kWh} / 720 \mathrm{kVA}$ Lithium-ion battery of EPFL.

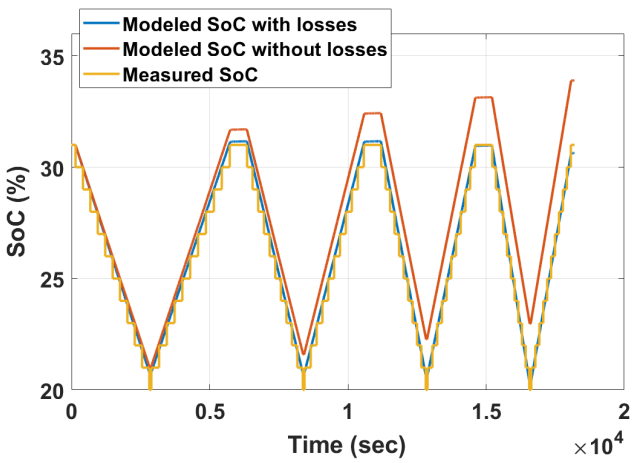

Figure 11: Measured vs modeled battery's SoC. 